\title{
AN OBSERVATIONAL EVIDENCE OF THE COMMON ENVELOPE PHASE
}

\author{
Marek J. Sarna ${ }^{1}$ and Jereny J. Drake ${ }^{2}$
}

RESUMEN

Se utilizaron por primera vez las observaciones de la binaria pre-cataclísmica V471 Tau, llevadas a cabo en el Espectrógrafo con Rejilla de Transmisión de Baja Energía de Chandra, para estimar el cocicnte de abundancia $\mathrm{C} / \mathrm{N}$ de la componente de la enana $\mathrm{K}$. Mientras que la componente de la cuana blanca domina el espectro más allá de $50 \AA$, en longitudes de onda más cortas la emisión observada de rayos X es debida por completo a la emisión coronal proveniente de la enana $K$. Las líneas de resonancia semejantes a $2 \mathrm{p}{ }^{2} \mathrm{P}_{3 / 2,1 / 2} \rightarrow 1 \mathrm{~s}{ }^{2} \mathrm{~S}_{1 / 2}$ de $\mathrm{C}$ y $\mathrm{N}$ proporcionan una estimación de su cociente de abundancia logarítmica respecto al Sol de $[\mathrm{C} / \mathrm{N}]=-0.38 \pm 0.15$. la mitad del valor solar actualmente aceptado. Este resultado lo interpretanos como la primera evidencia observacional clara de la presunta fase de envolvente común de este sistema durante la cual la superficie de la enana $\mathrm{K}$ fue contaminada por el material procesado por el ciclo $\mathrm{CN}$, que ha sido dragado dentro do la envolvente de la gigante roja.

\section{ABSTRACT}

Chandra Low Energy Transmission Grating Spectrograph observations of the pre-cataclysmic binary V471 Tau have been used to estimate the $\mathrm{C} / \mathrm{N}$ abundance ratio of the $\mathrm{K}$ dwari component for the first time. While the white dwarf component dominates the spectrum longward of $50 \AA$, at shorter wavelengths the observed X-ray emission is entirely due to coronal emission from the $\mathrm{K}$ dwarf. The H-like $2 \mathrm{p}^{2} \mathrm{P}_{3 / 2,1 / 2} \rightarrow 1 \mathrm{~s}^{2} \mathrm{~S}_{1 / 2}$ resonance lines of $\mathrm{C}$ and $\mathrm{N}$ yield an estimate of their logarithmic abundance ratio relative to the Sun of $[\mathrm{C} / \mathrm{N}]=-0.38 \pm 0.15$ half of the currently accepted solar value. We interpret this result as the first clear observational evidence for the presumed common envelope phase of this system, during which the surface of the $\mathrm{K}$ dwarf was contaminated by $\mathrm{CN}$-cycle processed material dredged up into the red giant envelope.

\section{Key Words: STARS: ABUNDANCES - STARS: ACTIVITY - STARS: CORONAE — X-RAYS: STARS}

\section{INTRODUCTION}

The eclipsing Hyades binary V471 Tauri lies at a distance of $47 \pm 3 \mathrm{pc}$ and comprises a cool mainsequence star (dK2) and a hot degenerate white dwarf (DA1.5) in an orbit with a period of 0.52 days. It is a detached binary in which neither star fills its Roche lobe, and has a separation of 3.1R. (Young \& Nelson 1972). The system is evolving toward a cataclysmic variable phase in which the $\mathrm{K}$ dwarf will overfill its Roche lobe and transfer mass through accretion onto the white dwarf. As such, V471 Tau is a rare example of a nearby pre-cataclysmic system and presents a valuable opportunity to study the origin and evolution this type of close binary. Drake \& Sarna (2003), using Chandra Low Energy Transmission Grating Spectrograph (LETGS) X-ray spectra of Ly $\alpha$ resonance transitions in $\mathrm{C}$ and $\mathrm{N} \mathrm{H}$-like ions, we determine for the first time the $\mathrm{C} / \mathrm{N}$ abundance ratio in the $\mathrm{K}$ dwarf secondary of V471 Tau. We

\footnotetext{
${ }^{1}$ Nicolaus Copernicus Astronomical Center, Warsaw, Poland.

${ }^{2}$ Smithsonian Astrophysical Observatory, Cambridge, USA.
}

show that the secondary star has half the $\mathrm{C} / \mathrm{N}$ ratio expected for the surface of an unevolved star, presenting the first definitive cvidence of the CE phase in which substantial surface contamination by the red giant envelope of its companion occurred.

\section{OBSERVATIONS AND ANALYSIS}

V471 Tau was observed for approximately one day by Chandra using the LETGS and High Resolution Camera (HRC) in standard instrument configurations, between 2002 January 24 UT 22:31 and January 25 UT 23:38. Observational data were obtained from the public Chandra Data Archive ${ }^{3}$, and pipeline-processed (CXC software version 6.3.1) photon event lists were reduced using the CIAO software package version 2.2 . The resulting spectra had an effective exposure time of $87494 \mathrm{~s}$. Spectra were analyzed using the PINTofALE IDL ${ }^{4}$ software suite (Kashyap \& Drake 2000).

The spectrum of V471 Tau is in the region between $23-36 \AA$ that contains the Ly $\alpha$ resonance

\footnotetext{
${ }_{3}$ http://asc.harvard.edu/cda

${ }^{4}$ Interactive Data Language, Research Systems Inc.
} 
lines of $\mathrm{H}$-like $\mathrm{N}$ and $\mathrm{C}$. For comparision we used the Chandra LETGS spectra of the K2 dwarf $\epsilon$ Eri and the KOIII giant $\beta$ Ceti obtained from the public archive and jrocessed in the same way as the V471 Tau observation.

The V471 Tau $\mathrm{C}$ and $\mathrm{N}$ lines are in a proportion intermediate between that of an unevolved composition exemplified by $\epsilon$ Eri and the post-dredge-up surface composition of $\beta$ Ceti. A formal value for the $\mathrm{C} / \mathrm{N}$ abundance ratio of V471 Tau is given by the observed spectral line flux ratio. The flux to abundance ratio conversion factor from Drake \& Sarna (2003), leads directly to the result $[\mathrm{C} / \mathrm{N}]=-0.38 \pm 0.15$ $([\mathrm{C} / \mathrm{N}]$ represents the logarithmic $\mathrm{C} / \mathrm{N}$ abundance zatio relative to the solar value).

\section{ACCRETION DURING THE CE PHASE}

During the CE phase, the red dwarf secondary effectively exists within the envelope of the red giant primary (Hjellming \& Taam 1991). The accreted material therefore has the chemical composition of a giant star. The material accreted during the $\mathrm{CE}$ phase will be mixed on a convective-mixing (dynamical) time-scale. The mass of the region that is mixed, $M_{m i x}$, consists of the sum of the masses of the convective region of the original star, $M_{\text {conv }}$, and the accreted matter $M_{a c c}$.

For the case of a typical intermediate mass star (2.5 $\left.M_{c}, Z=0.03\right)$, stellar evolutionary calculations predict a decrease in the surface $\mathrm{C} / \mathrm{N}$ abundance ratio between the zero age main-sequence and immediately prior to the first thermal pulse by a factor of 3.75 (e.g. Girardi et al. 1996). From Allende Prieto et al. $(2001,2002)$, the $\mathrm{C} / \mathrm{N}$ ratio for the Sun expressed in terms of mass fraction is 2.54 and the mass fractions of $\mathrm{C}$ and $\mathrm{N}$ are $X_{\odot}^{C}=0.00295$ and $X_{\odot}^{N}=0.00116$, respectively. The $\mathrm{C} / \mathrm{N}$ abundance by mass fraction at the surface of the red dwarf in the post-CE phase, $X_{r d}^{C} / X_{r d}^{N}$, is then given by

$$
\frac{X_{r d}^{C}}{X_{r d}^{N}}=\frac{M_{c o n n} X_{\odot}^{C}+M_{a c c} X_{g i a n t}^{C}}{M_{c o n v} X_{\odot}^{N}+M_{a c c} X_{g i a n t}^{N}} .
$$

The accreted mass can then be written

$$
M_{a c c}=M_{c o n v} \frac{X_{\odot}^{C}-\left(X_{r d}^{C} / X_{r d}^{N}\right) X_{\odot}^{N}}{\left(X_{r d}^{C} / X_{r d}^{N}\right) X_{\text {giant }}^{N}-X_{g i a n t}^{C}} .
$$

Here, the $\mathrm{C}$ and $\mathrm{N}$ mass fractions for the giant phase are assumed to be the solar values modified such that $X_{c, 0}^{C} / X_{i}^{N}$ is reduced by the factor of 3.75 predicted by evolutionary calculations, as noted above: $X_{\text {giant }}^{C}=0.00197, X_{\text {giant }}^{N}=0.0029$. The quantity $M_{a c c}$ is a function of the red dwarf initial mass. The convection zone mass, $M_{c o n v}$, has been taken from models computed using a standard stellar evolutionary code. The recent mass determination of O'Brien, Sion \& Bond (2001), $M=$ $0.93 \pm 0.07 M_{\odot}$, leads to an accreted mass in the range 0.011-0.029 $M_{\odot}$, and an initial convection zone mass of $M_{\text {conv }}=0.013-0.034 M_{\odot}$.

Sarna et al. (1995) described how accretion during the $\mathrm{CE}$ phase changes the ${ }^{12} \mathrm{C} /{ }^{13} \mathrm{C}$ ratio on the surface of the red dwarf. Based on the values of $M_{a c c}$ and $M_{\text {conv }}$ from above, we obtain a mass fraction ratio $X_{r d}^{12} / X_{r d}^{13}=30$. This value is slightly larger than the tentative estimate of $X_{r d}^{12} / X_{r d}^{13}=10$ based on $\mathrm{CO}$ bands near $2.35 \mu \mathrm{m}$ by Dhillon et al. (2002).

\section{CONCLUSIONS}

We have determined the $\mathrm{C} / \mathrm{N}$ abundance ratio in the corona of the $\mathrm{K}$ dwarf component of V471 Tau based on the resonance lines of $\mathrm{H}$-like $\mathrm{C}$ and $\mathrm{N}$ seen in Chandra LETGS spectra. The value we obtain, $\mathrm{C} / \mathrm{N}=0.1 \pm 0.15$, or $[\mathrm{C} / \mathrm{N}]=-0.38 \pm 0.15$, is intermediate between that of an unevolved surface composition and the surface composition of typical red giants. This result provides the most direct observational evidence to date of the CE phase of V471 Tau. Our $\mathrm{C} / \mathrm{N}$ abundance implies the $\mathrm{K}$ dwarf accreted $\sim 0.01-0.03 M_{\odot}$ from the envelope of the primary star during its red giant phase. This material was subsequently diluted by mixing with the convective envelope of the $\mathrm{K}$ dwarf. Our value for the accreted mass predicts a ${ }^{12} \mathrm{C} /{ }^{13} \mathrm{C}$ abundance ratio that is consistent with a recent observational estimate.

JJD was supported by NASA contract NAS839073 to the Chandra X-ray Center during the course of this research. MJS was supported through grant 5-P03D-004-21 of the Polish State Committee for Scientific Research.

\section{REFERENCES}

Allende Prieto, C., Berklem, P. S., Asplund, M., \& Ruiz Cobo, B. 2001, ApJ, 558, 830

Allende Prieto, C., Lambert, D. L., \& Asplund, M. 2002, ApJ, 573, L137

O'Brien, M. S., Bond, H. E., \& Sion, E. M. 2001, ApJ, 563,971

Dhillon, V. S., Littlefair, S. P., Marsh, T. R., Sarna, M. J., \& Boakes, E. H. 2002, A\&A, 393, 611

Drake, J.J., \& Sarna, M. J. 2003, ApJ, 594, L55

Girardi, L., Bressan, A., Bertelli, G., \& Chiosi, C. 2000, A\&AS, 141,371

Hjellming, M. S., \& Taam, R. E. 1991, ApJ, 370, 709

Kashyap, V., \& Drake, J.J. 2000, BASI, 28, 475

Sarna, M. J., Dhillon, V. S., Marsh, T. R. \& Marks, P. B. 1995, MNRAS, 272, L41

Young, A., \& Nelson, B. 1972, ApJ, 173, 653 